\title{
Assistência de enfermagem no parto humanizado
}

\author{
Nursing care in humanized delivery \\ Atención de enfermería en el parto humanizado
}

Núbia Rafaela Ferreira Da Costa Gomes ORCID: https://orcid.org/0000-0001-8488-2503

Universidade da Amazônia, Brasil

E-mail: rafaela1costagomes@gmail.com

Patriane Teixeira Martins Gouvea ORCID: https://orcid.org/0000-0002-0845-7174

Universidade da Amazônia, Brasil

E-mail: patrianemartinsgouvea@gmail.com

Octavio Augusto Barbosa Mendonça ORCID: https://orcid.org/0000-0001-8946-0238

Universidade da Amazônia, Brasil

E-mail: oabm73@yahoo.com.br

Rômulo Leno Miranda Barros

ORCID: https://orcid.org/0000-0003-1802-4521

Universidade da Amazônia, Brasil

E-mail: romuloleno@hotmail.com

Brenda Tanielle Dutra Barros

ORCID: https://orcid.org/0000-0002-3184-050X

Centro Universitário Metropolitano da Amazônia, Brasil

E-mail: brendatanielle.enf@gmail.com

Virgínia Mercês Lara Pessoa Oliveira

ORCID: https://orcid.org/0000-0002-4417-637X

Centro Universitário Metropolitano da Amazônia, Brasil

E-mail: virginiaoliveira@famaz.edu.br

Marcely Monteiro Da Silva

ORCID: https://orcid.org/0000-0001-5878-5531

Centro Universitário Metropolitano da Amazônia, Brasil

E-mail: marcelym98@gmail.com

Apolini Roberta De Figueiredo Dos Santos

ORCID: https://orcid.org/0000-0001-8097-4929

Escola Superior Madre Celeste, Brasil

E-mail: apoline-roberta@ hotmail.com

Fabiana De Souza Santos

ORCID: https://orcid.org/0000-0001-9591-6174

Escola Superior da Amazônia, Brasil

E-mail: fabianafisioterapeuta90@gmail.com

Thalia Paula Miranda Oliveira

ORCID: https://orcid.org/0000-0001-7949-7512

Escola Superior da Amazônia, Brasil

E-mail: thaliamiranda1426@gmail.com

Ruthe Dos Santos Da Silva

ORCID: https://orcid.org/0000-0002-3727-2494

Faculdade Estácio, Brasil

E-mail: ruth.santos155@gmail.com

Mivia Micaella Lima Reis

ORCID: https://orcid.org/0000-0003-1676-5844 Faculdade Estácio, Brasil

E-mail: miviareis@hotmail.com

José Eduardo Resende Campos

ORCID: https://orcid.org/0000-0003-1676-5844

Centro Universitário Metropolitano da Amazônia, Brasil

E-mail: educamposjrc@gmail.com

Ana Mara Franco Almeida Couto

ORCID: https://orcid.org/0000-0003-2871-6134

Faculdade Paraense de Ensino, Brasil

E-mail: maraanafranco@gmail.com

Tatiana Fabíola da Silva Lima

ORCID: https://orcid.org/0000-0001-5966-9727

Universidade do Estado do Pará, Brasil

E-mail: tatiana.fdslima@aluno.uepa.br 


\begin{abstract}
Resumo
Introdução: O parto humanizado possui várias vertentes que podem ser exploradas nas suas mais variadas dimensões, d formas que se integram entre si, visando promover parto e o nascimento saudável, bem como a prevenção da morbimortalidade através um conjunto de procedimentos e condutas adotadas. Objetivo: Evidenciar a assistência do enfermeiro na condução do parto humanizado. Metodologia: O estudo foi conduzido com base na pesquisa qualitativa, descritiva por meio de uma Revisão Integrativa da Literatura (RIL). O estudo foi realizado mediante pesquisa na Biblioteca Virtual em Saúde (BVS), acessando as bases de dados Literatura Latino-Americana e do Caribe em Ciências da Saúde (LILACS), Scientific Electronic Library Online (SCIELO), e Base de Dados de Enfermagem (BDENF), foi realizado em artigos científicos publicados e disponíveis em periódicos de 2015 a 2020. Discussão dos resultados: A partir dos critérios de inclusão e exclusão estabelecidos na metodologia, e 12 artigos foram selecionados para a revisão integrativa de literatura. A análise dos dados foi realizada a partir da Análise de Conteúdo de Bardin. Considerações finais: Para que haja uma assistência clínica de qualidade no processo do parto, é necessário a implementação de medidas que visem a redução da prática de métodos invasivos, o que poderá ser alcançado através da formação continuada dos profissionais que atuam nesta área, além da adequação do fator estrutural da instituição à parturiente. Desta forma, tem-se como resultado a prestação de cuidados de forma humanizada, acolhimento e satisfação do atendo ofertado.
\end{abstract}

Palavras-chave: Assistência; Enfermagem; Parto humanizado.

\begin{abstract}
Introduction: Humanized childbirth has several aspects that can be explored in its most varied dimensions, in ways that integrate with each other, aiming to promote healthy delivery and birth, as well as the prevention of morbidity and mortality through a set of adopted procedures and conducts. Objective: To demonstrate the assistance of nurses in conducting humanized childbirth. Methodology: The study was conducted based on qualitative research, descriptive through an Integrative Literature Review (RIL). The study was carried out through a search in the Virtual Health Library (VHL), accessing the Latin American and Caribbean Literature in Health Sciences (LILACS), Scientific Electronic Library Online (SCIELO), and Nursing Database (BDENF), was performed in scientific articles published and available in journals from 2015 to 2020. Discussion of results: Based on the inclusion and exclusion criteria established in the methodology, 12 articles were selected for the integrative literature review. Data analysis was performed using Bardin's Content Analysis. Final considerations: In order for there to be quality clinical care in the birth process, it is necessary to implement measures aimed at reducing the practice of invasive methods, which can be achieved through the continued training of professionals working in this area, in addition to adequacy of the institution's structural factor to the parturient. In this way, the result is the provision of care in a humanized way, welcoming and satisfying the service offered.
\end{abstract}

Keywords: Assistance; Nursing; Humanized birth.

\begin{abstract}
Resumen
Introducción: El parto humanizado tiene varios aspectos que pueden ser explorados en sus más variadas dimensiones, de manera que se integren entre sí, con el objetivo de promover el parto y el parto saludables, así como la prevención de la morbimortalidad a través de un conjunto de procedimientos y conductas adoptadas. Objetivo: Demostrar la asistencia del enfermero en la realización del parto humanizado. Metodología: El estudio se realizó con base en una investigación cualitativa, descriptiva a través de una Revisión de Literatura Integrativa (RIL). El estudio se realizó mediante una búsqueda en la Biblioteca Virtual en Salud (BVS), accediendo a la Literatura Latinoamericana y del Caribe en Ciencias de la Salud (LILACS), Biblioteca Electrónica Científica en Línea (SCIELO) y Base de Datos de Enfermería (BDENF), se realizó en artículos publicados y disponibles en revistas de 2015 a 2020. Discusión de resultados: A partir de los criterios de inclusión y exclusión establecidos en la metodología, se seleccionaron 12 artículos para la revisión integradora de la literatura. El análisis de datos se realizó mediante el análisis de contenido de Bardin. Consideraciones finales: Para que exista una atención clínica de calidad en el proceso del parto, es necesario implementar medidas encaminadas a reducir la práctica de métodos invasivos, lo cual se puede lograr mediante la formación continua de los profesionales que laboran en esta área, además de adecuación del factor estructural de la institución a la parturienta. De esta forma, el resultado es la prestación de cuidados de forma humanizada, acogiendo y satisfaciendo el servicio ofrecido.
\end{abstract}

Palabras clave: Asistencia; Enfermería; Nacimiento humanizado.

\title{
1. Introdução
}

A gravidez é um momento ímpar na vida da mulher; é o momento em que ocorrem muitas mudanças, o corpo dela se prepara para gerar um novo ser e um turbilhão de sentimentos aparece nessa fase da vida. São dúvidas, medos que irão levar a mulher a uma instabilidade emocional, e é nesse momento que o apoio incondicional da família e das pessoas que irão acompanhar esta gravidez, é indispensável. A atenção voltada a essa gestante deve ser diferenciada, respeitando suas 
particularidades e seu tempo, isso deve acontecer desde as consultas de pré-natal até o momento do parto, momento em que se inicia o atendimento humanizado (Moura et al., 2017).

O termo humanização acarreta um amplo conceito, tendo início desde o acolhimento da gestante durante o acompanhamento do pré-natal até as boas práticas de atendimento proporcionado pela equipe de saúde, buscando evitar intervenções desnecessárias e fornecendo um trabalho de parto saudável. A assistência prestada durante o parto deve ocorrer de uma forma humanizada, respeitando e criando condições para que todas as dimensões espirituais, psicológicas, físicas do ser humano sejam alcançadas no momento do parto. Observar o paciente de forma holística significa humanizar a assistência, pois se consegue ofertar cuidados de forma integral e equitativa, gerando uma concepção assertiva sobre o processo saúde-doença (Ferreira et al., 2018).

O enfermeiro é responsável pelo acompanhamento às gestantes durante o pré-natal, de modo a garantir atendimento por meio das consultas de enfermagem, avaliando as condições de saúde materna, clínica e obstétricas, integralmente (Brasil, 2019). As ações do enfermeiro na atenção básica à saúde são pautadas em práticas de cuidado seguras, eficientes e de alta qualidade, que fortalecem os serviços de atenção básica, distantes do modelo biomédico, trazendo uma perspectiva de assistências ampliada e integral das necessidades e demandas de saúde do indivíduo, família e coletividade (Fertonani et al., 2015).

Para a realização do parto humanizado deve-se priorizar a autonomia da mulher, emponderando-a através da oferta de informações esclarecedoras sobre os procedimentos e o processo do parto, no intuito, também, de minimizar as intervenções invasivas; desta forma, contribuindo para que o parto ocorra de forma natural, sem distocia e com um vínculo de confiança estabelecido entre a equipe e a parturiente (Santana et al., 2019).

Para que ocorra esta independência, uma relação de confiança deve ser estabelecida entre a enfermagem e a gestante, desde o início da gestação, passando pelo parto e puerpério, criando um vínculo afetivo de confiança, respeito e pautado no diálogo efetivo. É atribuição do enfermeiro o planejamento, coordenação e avaliação da qualidade dos serviços prestados pela equipe de enfermagem, principalmente no que tange aos cuidados com o binômio mãe-bebê, sendo necessário a ação do trabalho interdisciplinar para que não haja ocorrências negativas (Ferreira et al., 2018).

O Brasil está na lista dos países recordistas em partos cesarianos no mundo. Anualmente, pelo Sistema Único de Saúde (SUS) são realizados 2,3 milhões de partos e $80 \%$ destes são cirúrgicos, fato preocupante tendo em vista que a Organização Mundial de Saúde (OMS) recomenda uma taxa aceitável entre 10 e $15 \%$. Diante desses fatos o ministério da saúde preconiza o parto humanizado, que pode ser realizado em Centros de Parto Normal (CPN) ou mesmo no domicílio da parturiente (Moura et al., 2017).

A falta de humanização no trabalho de parto acarreta várias situações negativas, como violência obstétrica, que pode ser indiciada no pré-natal quando é oferecida gestantes informações insuficiente ou que essas não estão seguras para o mandamento da sua gestação dessa forma a gestação é induzida a escolher um parto cirúrgico cesárea sem relevâncias clínicas evidentes (Leal, 2018).

O papel do profissional de enfermagem, nesse contexto, começa na assistência à saúde da mulher e do recém-nascido, buscando garantir uma atenção humanizada, individualizada e ampla. Além disso, também cabe ao enfermeiro promover ações que visem a atender a parturiente em sua complexidade, possibilitando sua autonomia quanto aos assuntos relativos aos cuidados no pré-natal, parto, primeiros cuidados com o bebê e puerpério (Camillo et al., 2016).

A oferta de uma assistência pautada na humanização do acompanhamento da gestante, deste o início da gravidez até parto e pós-parto, também são competências da enfermagem. Por serem os profissionais que têm contado de maior frequência com a parturiente, a equipe de enfermagem acaba assumindo um papel decisivo no cuidado a esta paciente. Desta forma, é de suma importância que o profissional de enfermagem desenvolva habilidades e competências pautadas na ciência e subsidiada 
por instrumentos pertinentes e educação permanente, no intuito de tornar-se consciente do seu papel como agente de transformação (Nascimento et al., 2018).

Partindo desse pressuposto é importante a participação dos enfermeiros obstetras pois é competência da equipe de enfermagem oferecer uma assistência que proporcione o suporte necessário e continuo para que o parto seja um processo saudável e natural para que não venha se tornar uma experiência desagradável. É inegável que O profissional de enfermagem tem papel essencial no apoio ao parto humanizado, ofertando dados pertinentes à parturiente sobre a evolução do seu parto. Considerando-se o exposto, o estudo objetivou evidenciar a assistência do enfermeiro na condução do parto humanizado em publicações científicas, no período de 2015 - 2020.

Sendo assim, o presente estudo fortalecerá com as evidências para a formação de estratégias de fomento ao enfermeiro para alcançar boas práticas assistenciais que iniciam durante o pré-natal, sobrepondo o modelo biomédico, visando a promoção, no momento do parto, tornando-se assim um momento único, feliz e humanizado. Além de contribuir positivamente com o meio acadêmico na formação de conhecimento e produção cientifica (Leal et al., 2018).

\section{Metodologia}

O estudo foi conduzido com base na pesquisa qualitativa, descritiva por meio de uma Revisão Integrativa da Literatura (RIL).

O estudo foi realizado mediante pesquisa na Biblioteca Virtual em Saúde (BVS), acessando as bases de dados Literatura Latino-Americana e do Caribe em Ciências da Saúde (LILACS), Scientific Electronic Library Online (SCIELO), e Base de Dados de Enfermagem (BDENF), de acordo com o que foi disponibilizado utilizando os descritores selecionados. No período de agosto a outubro de 2021, respeitando o cronograma da pesquisa.

O estudo foi realizado em artigos científicos publicados e disponíveis em periódicos de 2015 a 2020.

Foram incluídas: Pesquisas em formatos artigos; Publicações que correspondam aos últimos 5 anos; Publicações no idioma português; Publicações de periódicos nacionais. Foram excluídos: Publicações que não estão completas e na íntegra de forma gratuitas em meio eletrônico; Tese, Dissertação, RIL e Realise; Trabalhos publicados por profissionais que não sejam da área da saúde; Anuários duplicados.

Esta pesquisa foi realizada em 6 etapas: 1) Determinação do tema e seleção da questão de pesquisa; 2) Estabelecimento dos critérios de inclusão e exclusão para a pesquisa e busca na literatura; 3) Definição das informações extraídas das pesquisas selecionadas; 4) Avaliação das pesquisas incluídas; 5) Interpretação dos resultados, 6) Revisão/síntese do conhecimento (Ercole et al., 2014).

Nesse sentido, a questão que norteou a busca foi: "Como é a assitencia prestada pelo enfermeiro na condução do parto evidenciados na literatura?"

A coleta de dados seguiu com base na presente questão, bem como no que se refere aos critérios de inclusão e exclusão.

Foi realizada uma pesquisa nas bases de dados por meio do cruzamento dos Descritores em Ciências da saúde (DeCS): Assistência; Enfermagem; Parto humanizado; utilizando o operador booleano AND para conjuga-los.

Dessa maneira, os critérios primários de inclusão, como período analisado; idioma; tipo de estudo; bases de dados e artigos com resumo e que estejam disponíveis de modo completo, serão aplicados sob o número total de estudos encontrados, sendo estes responsáveis pelo primeiro filtro dentre as pesquisas. Após isso, o número de artigos que figurarem foram analisados conforme a leitura dos títulos e resumos, nessa etapa permanecerão os artigos que contemplem, com as informações iniciais dispostas nas seções analisadas os conteúdos condizentes aos objetivos desta RIL. 
Seguindo a coleta, os estudos selecionados seguiram para a etapa de leitura na íntegra, onde se buscou identificar se os artigos que responderam à questão norteadora desta pesquisa.

Em relação aos estudos que apresentaram evidências científicas pertinentes, foram coletados os dados dos artigos selecionados. Vale ressaltar que todas essas etapas, bem como a exposição dos dados armazenados no banco de dados foram dispostos por meio de fluxograma e quadros, respectivamente.

A coleta de dados foi realizada por meio de um formulário (Ursi adaptado, 2005), onde nele constam itens que sintetizam as informações dos artigos encontrados. Cada item corresponde a uma informação extraída do artigo, a exemplo do título do artigo, autores, tipo de estudo, abordagem e entre outras.

Este estudo respeitou os direitos autorais dos autores consultados, utilizando as normas da Associação Brasileira de Normas Técnicas (ABNT) para citações e referências.

Essa pesquisa teve como benefício acrescentar à literatura dados referentes ao tema, assim como incentivar os profissionais enfermeiros a proporcionar uma condução adequada ao parto humanizado de forma equânime e integral.

A análise dos dados foi realizada por meio da técnica de análise de conteúdo de Bardin (1977) no qual se caracteriza como métodos e técnicas específicas para elaboração esquemática. Desse modo, os estudos analisados foram segregados em eixos temáticos. Além do supracitado, foi realizada a análise descritiva qualitativa, avaliando o contexto de que possíveis resultados serão mensurados. Para tanto foi realizada uma leitura minuciosa dos artigos para obter resposta sobre o problema da pesquisa, visando assim discutir e comparar os resultados coletados nas revisões bibliográficas com o intuído de confirmar os objetivos da pesquisa.

\section{Resultados e Discussão}

A partir dos critérios de inclusão e exclusão estabelecidos na metodologia, foram encontrados 1.318 artigos, sendo que: 714 eram resumos, dissertações, teses, 117 publicados em língua estrangeira, 268 eram fora do recorte temporal estabelecido, e 219 artigos estavam duplicados nas bases de dados, restando assim 12 artigos selecionados para a revisão integrativa de literatura, conforme descrito no fluxograma de amostragem. 
Figura 1 - Fluxograma de amostragem.

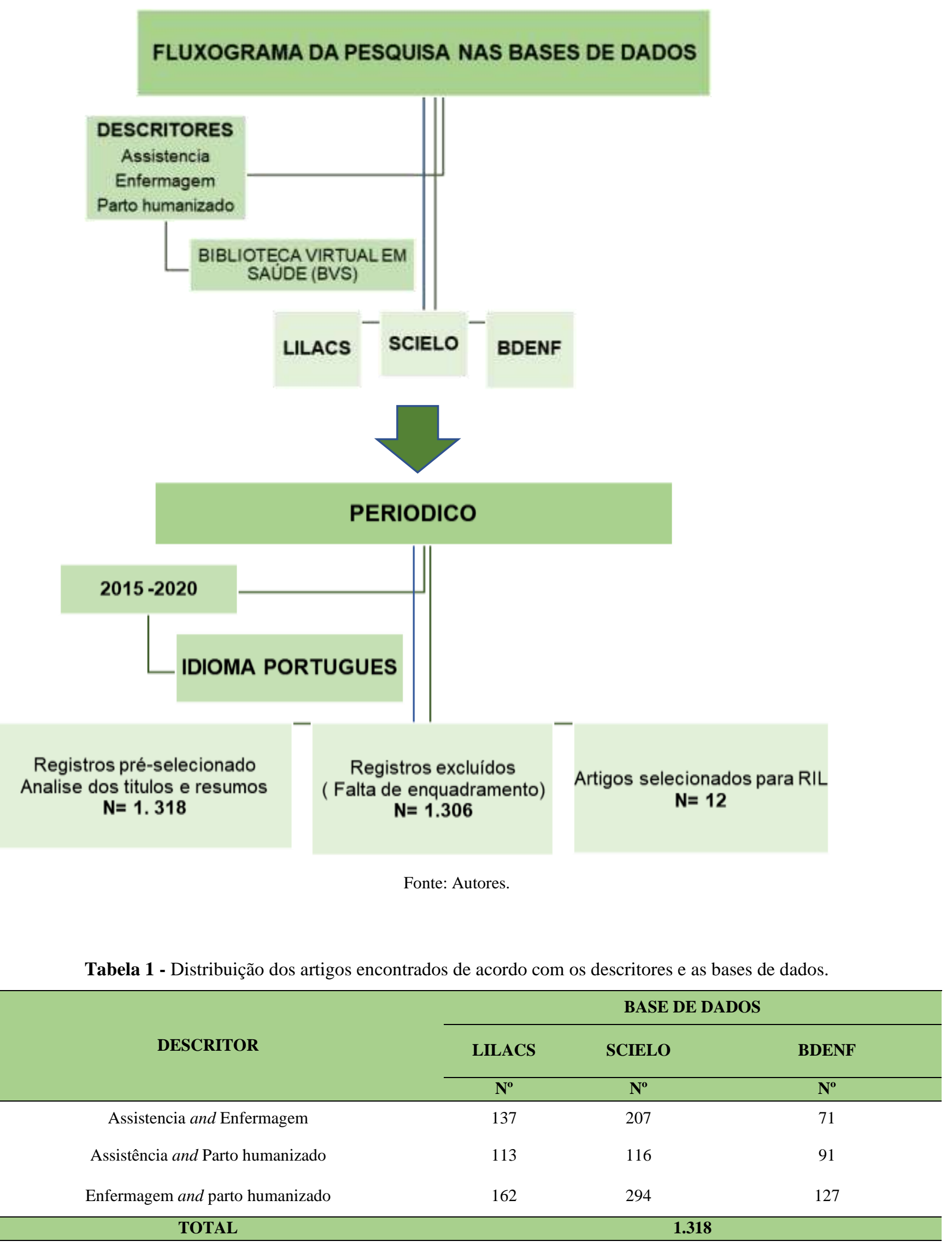

Fonte: Autores. 
Tabela 2 - Distribuição dos artigos encontrados de acordo com as bases de dados.

\begin{tabular}{|c|c|c|c|}
\hline \multirow{3}{*}{ ARTIGOS } & \multicolumn{3}{|c|}{ BASE DE DADOS } \\
\hline & LILACS & SCIELO & BDENF \\
\hline & $\mathbf{N}^{\circ}$ & $\mathbf{N}^{\circ}$ & $\mathbf{N}^{0}$ \\
\hline ENCONTRADOS & 412 & 617 & 289 \\
\hline INCLUÍDOS & 4 & 6 & 2 \\
\hline
\end{tabular}

Fonte: Autores.

De acordo com os dados das Tabelas 1 e 2 verifica-se que, dos 1.318 artigos encontrados, apenas 12 , sendo que 50\% (N: 06) foram selecionados na base de dados SCIELO, $17 \%$ (N: 02) na BVS/BDENF e 33\% (N: 04) no LILACS.

Organizou-se a integração das evidências dos artigos selecionados, para tal elaborou-se quadro síntese 1, que possibilitou a análise das informações e categorização dos conteúdos analisados, apresentado a seguir:

Quadro 1 - Distribuição dos artigos incluídos no estudo de acordo com os autores, as bases de dados e ano de publicação

\begin{tabular}{|c|c|c|c|c|}
\hline $\mathbf{N}^{\mathbf{0}}$ & ARTIGO & AUTORES & $\begin{array}{l}\text { BASE DE } \\
\text { DADOS }\end{array}$ & $\begin{array}{c}\text { ANO DE } \\
\text { PUBLICAÇÃO }\end{array}$ \\
\hline A1 & $\begin{array}{l}\text { Práticas de assistência ao parto normal: formação na } \\
\text { modalidade de residência }\end{array}$ & $\begin{array}{l}\text { Santos AHL dos, Nicácio MC, Pereira } \\
\text { ALF et al. }\end{array}$ & SCIELO & 2017 \\
\hline A2 & $\begin{array}{l}\text { Humanização do parto: significados e percepções de } \\
\text { enfermeiras }\end{array}$ & $\begin{array}{l}\text { Possati AB, Prates LA, Cremonese L, } \\
\text { Scarton J, Alves CN, Ressel LB }\end{array}$ & SCIELO & 2017 \\
\hline A3 & $\begin{array}{l}\text { Análise de partos acompanhados por enfermeiras } \\
\text { obstétricas na perspectiva da desmedicalização }\end{array}$ & $\begin{array}{l}\text { Reis, C. R. C; Silva, A. C. V; Souza, } \\
\text { D. O. M; Progianti, J. M; Vargens, O. } \\
\text { M. C. }\end{array}$ & LILACS & 2016 \\
\hline A4 & $\begin{array}{l}\text { Boas Práticas na atenção obstétrica e sua interface com a } \\
\text { humanização da assistência }\end{array}$ & $\begin{array}{l}\text { Andrade; L. F. B; Rodrigues; Q. P; } \\
\text { Silva, R. C. V. }\end{array}$ & BENDF & 2017 \\
\hline A5 & $\begin{array}{l}\text { Manejo não farmacológico de alívio da dor em partos } \\
\text { assistidos por enfermeira obstétrica }\end{array}$ & $\begin{array}{l}\text { Lehugeur D, Strapasson MR, Fronza } \\
\text { E. }\end{array}$ & SCIELO & 2017 \\
\hline A6 & $\begin{array}{l}\text { O cuidado de enfermeiras de um programa de residência } \\
\text { obstétrica sob o olhar da humanização. }\end{array}$ & $\begin{array}{l}\text { Giantaglia FN, Garcia ESGF, Rocha } \\
\text { LCT da et al. }\end{array}$ & LILACS & 2017 \\
\hline A7 & $\begin{array}{l}\text { Grupos de gestantes: espaço para humanização do parto e } \\
\text { nascimento }\end{array}$ & $\begin{array}{l}\text { Matos GC; Demori CC; Escobal APL; } \\
\text { et al. }\end{array}$ & SCIELO & 2017 \\
\hline A8 & $\begin{array}{l}\text { Atuação da enfermeira obstetra no desenrolar do trabalho } \\
\text { de parto e parto }\end{array}$ & $\begin{array}{l}\text { Alves, T. T. M; Paixão, G. P. N; } \\
\text { Fraga, C. D. S; Lírio, J. G. S; Oliveira, } \\
\text { F. A. }\end{array}$ & BDENF & 2018 \\
\hline A9 & $\begin{array}{l}\text { Boas práticas de atenção ao parto e ao nascimento na } \\
\text { perspectiva de profissionais de saúde }\end{array}$ & $\begin{array}{l}\text { Pereira SB, Diaz CMG, Backes MTS, } \\
\text { Ferreira CLL, Backes DS. }\end{array}$ & LILACS & 2018 \\
\hline A10 & $\begin{array}{l}\text { Implementação das práticas baseadas em evidências na } \\
\text { assistência ao parto normal }\end{array}$ & $\begin{array}{l}\text { Côrtes, CT; Oliveira, SMJV; Santos, } \\
\text { RCS; Francisco, AA; Riesco, MLG; } \\
\text { Shimoda, GT. }\end{array}$ & SCIELO & 2018 \\
\hline A11 & $\begin{array}{l}\text { Implementação das boas práticas na atenção ao parto em } \\
\text { Maternidade de referência }\end{array}$ & $\begin{array}{l}\text { de Melo, B. M; Gomes, L. F. S; } \\
\text { Henriques, A. C. P. T; Lima, S. K. M; } \\
\text { Damasceno, A. K. C }\end{array}$ & SCIELO & 2017 \\
\hline A12 & $\begin{array}{l}\text { À margem da humanização? Experiências de parto de } \\
\text { usuárias de uma maternidade pública de Porto Alegre-RS }\end{array}$ & Pedroso, C. N. L. S; López, L. C. & LILACS & 2017 \\
\hline
\end{tabular}

Fonte: Autores.

De acordo com os dados do Quadro 1, observasse-se a distribuição dos artigos incluídos no estudo de acordo com o periódico, os autores, as bases de dados e ano de publicação. 
Quadro 2 - Distribuição dos artigos incluídos no estudo de acordo com o objetivo e os achados da pesquisa sobre o papel do enfermeiro na condução do parto humanizado.

\begin{tabular}{|c|c|}
\hline $\mathbf{N}^{\mathbf{o}}$ & ACHADOS DA PESQUISA \\
\hline A1 & $\begin{array}{l}\text { Dentre as práticas úteis e que devem ser estimuladas, a OMS inclui os métodos não invasivos e não farmacológicos de alívio da } \\
\text { dor, como massagem e técnicas de relaxamento, durante o trabalho de parto. }\end{array}$ \\
\hline $\mathrm{A} 2$ & $\begin{array}{l}\text { Destaca o papel do enfermeiro quanto o acolhimento que compreende um momento oportuno para que a equipe de saúde possa } \\
\text { demonstrar atenção, interesse e disponibilidade, buscando conhecer e compreender as expectativas da parturiente e sua família, } \\
\text { esclarecendo as dúvidas relacionadas à gestação e ao parto. }\end{array}$ \\
\hline A3 & $\begin{array}{l}\text { Compreende a importância de humanizar e qualificar a atenção à gestante está garantindo a qualidade na assistência, o que } \\
\text { repercute em melhores resultados obstétricos, com uma postura acolhedora, respeitosa, os profissionais atendem às expectativas } \\
\text { e necessidades da mulher. }\end{array}$ \\
\hline A4 & $\begin{array}{l}\text { Em relação aos métodos não farmacológicos para o alívio da dor, eles devem ser oferecidos às parturientes desde sua admissão } \\
\text { na unidade para uma melhor condução do trabalho de parto, e melhores resultados perinatais. Sua utilização é benéfica, na } \\
\text { medida em que oferece alternativa e medidas de conforto, melhorando a assistência ao parto. }\end{array}$ \\
\hline A5 & $\begin{array}{l}\text { Está inserida na assistência ao parto de risco habitual e, em sua prática assistencial, busca implementar o manejo da dor, } \\
\text { caracterizando um modelo de cuidado centrado na fisiologia do parto e no protagonismo da mulher. }\end{array}$ \\
\hline A6 & $\begin{array}{l}\text { A importância de o profissional depositar segurança por meio da assistência qualificada, orientando que a dor que a mulher está } \\
\text { sentindo é fisiológica e normal e que desse modo ela saiba e confie que é capaz de passar por todo o processo do parto e } \\
\text { nascimento da melhor maneira possível. }\end{array}$ \\
\hline A7 & $\begin{array}{l}\text { Dispor de condições para que a parturiente tenha melhor tolerância à dor e ao desconforto, buscando reduzir os fatores que } \\
\text { aumentam as dores com a utilização de métodos mais eficientes. }\end{array}$ \\
\hline A8 & $\begin{array}{l}\text { Ressaltaram a importância do acolhimento para que o parto possa fluir bem, esperando-se que assim ocorra a redução de } \\
\text { intercorrências. Citaram também sobre a problemática do trabalho em equipe e do amparo para com a família, que muitas vezes } \\
\text { se torna uma tarefa difícil, principalmente quando as notícias não ocorrem como se espera. }\end{array}$ \\
\hline A9 & $\begin{array}{l}\text { Sob esse movimento circular e dinâmico em que a mulher é a protagonista. Dentre as práticas ineficazes ou prejudiciais na } \\
\text { condução do parto normal e que precisam ser desencorajadas, apresentam-se a utilização do enema, a tricotomia, a cateterização } \\
\text { profilática de rotina, a manobra de Valsalva durante o segundo estágio do trabalho de parto, a manobra de distensão perineal, } \\
\text { dentre outras. }\end{array}$ \\
\hline A10 & $\begin{array}{l}\text { A pratica das técnicas não farmacológicas para alívio da dor, o respeito à escolha da melhor posição para parir influencia } \\
\text { positivamente na evolução do trabalho de parto. }\end{array}$ \\
\hline A11 & $\begin{array}{l}\text { Referente à dor no parto, forma de alívio pode ser necessária. As massagens corporais, banhos (de chuveiro ou imersão), } \\
\text { técnicas de respiração e relaxamento, deambulação ativa, toques confortantes, utilização das bolas e cavalinhos, exercícios e } \\
\text { outras medidas de suporte físico e emocional devem ser utilizadas para alívio da dor. }\end{array}$ \\
\hline A12 & $\begin{array}{l}\text { O empoderamento da mulher durante a assistência deve ser estimulado, de forma que a autonomia em tomar decisões referente a } \\
\text { sua saúde, influencie de forma positiva na qualidade dos serviços oferecidos }\end{array}$ \\
\hline
\end{tabular}

Fonte: Autores.

De acordo com os dados do Quadro 2, demostram uma desarmonia em relação a prática e teoria do parto humanizado. Entre as transformações vistas nas pesquisas realizadas encontramos: mudança no ambiente: acolhedor, limpo e confortável; presença de familiares; oferta de medidas de alívio da dor; menos as medidas invasivas. Muito relevante para a enfermagem é a normatização, sistematização e implantação de regras para efetivar na prática o parto humanizado.

Portanto, os profissionais de enfermagem devem interagir com os pacientes para entender o que está acontecendo. Desenvolver uma comunicação clara evitará a desinformação dos pacientes, pois a inserção deste leva a uma deficiência no cuidado (Castro \& Krapis, 2019).

Não existem dúvidas quanto à necessidade de incorporação universal e integral do parto humanizado para benefício da mulher e criança. Apesar de ainda haver número elevado de realização de partos cirúrgicos sem indicação, o que contraria um dos princípios iniciais e necessário para considerar que o parto foi humanizado e por esse e outros fatores não se chegou aos números ideais dessa política (Alves et al., 2018).

Durante a admissão da paciente, o risco obstétrico deve ser avaliado, isto determinará a conduta que deve ser adotada no atendimento a esta usuária. No momento da anamnese ou exame físico, já possível visualizar sinais que demonstram a inviabilização do parto vaginal, a exemplo, apresentação atípica do feto, principalmente na gravidez gemelar, gestantes 
portadoras HIV, hepatite $\mathrm{C}$ ou outras infecções, e apresentação de cicatriz longitudinal no útero, decorrente de cesariana anterior (Comissão Nacional De Incorporação De Tecnologias No Sistema Único De Saúde, 2016).

Após análise deste o risco, o enfermeiro programa condutas (ou práticas humanizadas) a serem adotadas como, prescrição de um plano de ações assistenciais ao parto. Com a possibilidade de distocias descartadas, o enfermeiro vê a necessidade de não permitir a restrição alimentar da gestante, visto que o momento do parto é o que mais exige energia física da mulher (Santos et al., 2017).

Entende-se por práticas humanizadas, práticas que não envolvam o uso de fármacos no alívio da dor, a exemplo: massagens, exercícios respiratórios, banho com água morna, permissão de um acompanhante, promoção da autonomia da mulher, escolha de posição, tomadas de decisões em conjunto (equipe, parturiente e acompanhante) sobre as decisões que envolvem (Alves et al., 2018). A seguir, aborda-se a distribuição destes artigos incluídos no estudo:

Quadro 3 - Distribuição dos artigos incluídos no estudo de acordo com o objetivo e os achados da pesquisa sobre como assistência de enfermagem contribui através das práticas do parto humanizado.

\begin{tabular}{|c|c|}
\hline $\mathbf{N}^{\mathbf{o}}$ & ACHADOS DA PESQUISA \\
\hline A1 & $\begin{array}{l}\text { Humanização do parto pressupõe a atenção centrada na mulher, incentivando a substituição de práticas intervencionistas por } \\
\text { práticas menos invasivas e direcionando os cuidados a partir de condutas mais humanizadas. }\end{array}$ \\
\hline $\mathrm{A} 2$ & $\begin{array}{l}\text { Enfatizaram a importância de a mulher ter autonomia para a livre movimentação durante o trabalho de parto. Pondera-se que esta } \\
\text { é uma prática que favorece a progressão do feto, mas que também segue os desígnios corporais femininos e proporciona maior } \\
\text { conforto à parturiente. }\end{array}$ \\
\hline A3 & $\begin{array}{l}\text { O enfermeiro oferecendo as parturientes à oportunidade de vivenciar seu parto numa abordagem desmedicalizada e humanizada. } \\
\text { Isto foi verificado com a alta incidência da oferta de tecnologias não invasivas de cuidado. }\end{array}$ \\
\hline A4 & Orientar as gestantes sobre os benefícios das boas práticas e de suas contribuições para uma boa condução do parto e pós-parto. \\
\hline A5 & $\begin{array}{l}\text { Prática assistencial, busca implementar o manejo da dor, caracterizando um modelo de cuidado centrado na fisiologia do parto e } \\
\text { no protagonismo da mulher. }\end{array}$ \\
\hline A6 & $\begin{array}{l}\text { A humanização da assistência durante o processo de parto-nascimento inclui principalmente que a enfermeira obstetra respeite os } \\
\text { aspectos da fisiologia da mulher, sendo essencial que cuidados não farmacológicos de alívio da dor sejam explorados, por serem } \\
\text { mais seguros e evitarem a necessidade de tantas intervenções. }\end{array}$ \\
\hline A7 & $\begin{array}{l}\text { Humanizar o parto é dar às mulheres o direito a um atendimento focado em suas necessidades, respeitando a sua individualidade e } \\
\text { seus desejos. }\end{array}$ \\
\hline A8 & $\begin{array}{l}\text { Compreende a importância de humanizar e qualificar a atenção à gestante está garantindo a qualidade na assistência, o que } \\
\text { repercute em melhores resultados obstétricos, com uma postura acolhedora, respeitosa, os profissionais atendem às expectativas e } \\
\text { necessidades da mulher. }\end{array}$ \\
\hline A9 & $\begin{array}{l}\text { Dentre as boas práticas, também foi referida a presença de acompanhante de escolha da mulher no momento do parto e do } \\
\text { nascimento. }\end{array}$ \\
\hline A10 & $\begin{array}{l}\text { A prática que apresentou diminuição estatística foi prescrição/administração de ocitocina no trabalho de parto. A análise dos } \\
\text { prontuários mostrou redução estatística de amniotomia e da posição litotômica, com consequente aumento das posições } \\
\text { verticais/cócoras. }\end{array}$ \\
\hline A11 & $\begin{array}{l}\text { O acompanhamento da evolução do trabalho de parto deve ser feito por meio do partograma para que seja possível diagnosticar as } \\
\text { possíveis alterações e indicar a tomada de condutas apropriadas para a sua resolução }\end{array}$ \\
\hline A12 & $\begin{array}{l}\text { Práticas tecnocráticas de atenção ao parto. Persiste o uso rotineiro de intervenções obstétricas, como a amniotomia e a indução do } \\
\text { parto por ocitocina sintética. }\end{array}$ \\
\hline
\end{tabular}

Fonte: Autores.

De acordo com os dados do quadro 3, verifica-se sobre o papel do enfermeiro na condução do parto humanizado que consistem em práticas que não fazem utilização de recursos farmacológicos para aliviar a dor (Alves et al., 2018; Brasil, 2019).

Uma abordagem humanizada irá incluir o fornecimento do apoio integral, de forma transversal, à gestante e sua família. Isto inclui a assistência desde o pré-natal até o momento da concepção do bebê, sendo esta instruída sobre a alimentação adequada, sobre a deambulação no momento das contrações e o empoderamento desta (Melo et al., 2017).

$\mathrm{O}$ enfermeiro assume o papel de agente executor de uma assistência qualificada, que torne o momento do parto seguro, com minimização dos incômodos gerados, tentando torna-lo um ato mais prazeroso do que doloroso, pois este é o 
nascimento de um filho e de uma nova família; é o momento que acarreta grandes transformações sociais (Andrade et al., 2017).

Se a equipe não ofertar um manuseio adequado, a vivência do parto poderá trazer traumas, o que aumentam possibilidade de distorcias obstétricas, entretanto condutas realizadas para amenizar e controlar a dor do parto também é um papel fundamental do enfermeiro durante a pratica do parto humanizado (Andrade et al., 2017).

Assumir uma postura que transfira calma e segurança, dá à parturiente possibilidades de se desenvolver melhor, no sentido do prosseguimento ao parto normal. Palavras de incentivo aumentam sua confiança dar continuidade com naturalidade o parto (Cortês et al., 2018).

Quadro 4 - Distribuição dos artigos incluídos no estudo de acordo com o objetivo e os achados da pesquisa sobre os tipos de abordagens metodológicas durante o parto.

\begin{tabular}{|c|c|}
\hline $\mathbf{N}^{\mathbf{0}}$ & ACHADOS DA PESQUISA \\
\hline A1 & $\begin{array}{l}\text { O uso da água por meio do banho morno de aspersão, das técnicas de respiração e da massagem promovem a redução da dor e o } \\
\text { conforto da parturiente. Inclui-se a posição supina durante o trabalho de parto entre as práticas claramente prejudiciais ou } \\
\text { ineficazes e que devem ser eliminadas. }\end{array}$ \\
\hline A2 & $\begin{array}{l}\text { Utilização de técnicas de conforto da dor e a participação ativa da mulher são práticas vinculadas à humanização do parto. Essas } \\
\text { práticas tendem a contribuir para que a parturiente tenha mais liberdade e autonomia }\end{array}$ \\
\hline A3 & $\begin{array}{l}\text { A oportunidade de vivenciar seu parto numa abordagem desmedicalizada e humanizada. Outras tecnologias não invasivas de } \\
\text { cuidado empregadas portanto, na perspectiva desmedicalizadora, foram: a presença do acompanhante, constatada dos trabalhos de } \\
\text { partos e/ou partos; os exercícios respiratórios o estímulo à deambulação em e à liberdade para movimentos pélvicos em o uso da } \\
\text { água através do banho em a realização de massagens. }\end{array}$ \\
\hline A4 & $\begin{array}{l}\text { Nessa perspectiva, as boas práticas elencadas para este estudo presença de acompanhante, métodos não farmacológicos para o } \\
\text { alívio da dor, liberdade de escolha da posição, contato pele a pele imediato e amamentação na sala de parto. }\end{array}$ \\
\hline A5 & $\begin{array}{l}\text { O manejo da dor contempla uma série de práticas complementares e métodos não farmacológicos, como: banho de imersão ou } \\
\text { aspersão, bola suíça, aromaterapia, musicoterapia, acupuntura, massagem, suporte emocional contínuo, verticalização da mulher e } \\
\text { variedade de posição, entre outros. A dor durante o trabalho de parto pode levar à perda do controle emocional da mulher, } \\
\text { constituindo um evento estressor e traumático capaz de levar a desordens mentais. }\end{array}$ \\
\hline A6 & $\begin{array}{l}\text { Inclui principalmente que a enfermeira obstetra respeite os aspectos da fisiologia da mulher, sendo essencial que cuidados não } \\
\text { farmacológicos de alívio da dor sejam explorados, por serem mais seguros e evitarem a necessidade de tantas intervençoses. }\end{array}$ \\
\hline A7 & $\begin{array}{l}\text { Entre os métodos utilizados pelas puérperas entrevistadas os mais citados foram: massagem (proporciona uma estimulação } \\
\text { sensorial causando diminuição do estresse emocional e aumentando o fluxo sanguíneo bem como a oxigenaçãa) banho no } \\
\text { chuveiro (este deve ser de preferência aquecido o que promove a vasodilatação periférica, redistribuição do fluxo sanguíneo } \\
\text { consequentemente relaxamento muscular). }\end{array}$ \\
\hline A8 & $\begin{array}{l}\text { Salienta-se que o principal entendimento sobre o termo 'humanizar' é o de 'acolher' a mulher, dando-lhe toda autonomia que } \\
\text { precisa. }\end{array}$ \\
\hline A9 & Dentre as boas práticas, foi referida a presença de acompanhante de escolha da mulher no momento do parto e do nascimento. \\
\hline A10 & $\begin{array}{l}\text { A assistência realizada com métodos não farmacológicos e não invasivos contribuem positivamente para a efetivação do parto. } \\
\text { Entre eles destaca-se a utilização do banho de chuveiro, da bola suíça, do uso do cavalinho, das barras, da deambulação e das } \\
\text { massagens são benéficos para alívio das contrações e relaxamento da mulher, além de auxiliar na dilatação e expulsão do recém- } \\
\text { nascido. }\end{array}$ \\
\hline A11 & $\begin{array}{l}\text { O acompanhamento da evolução do trabalho de parto deve ser feito por meio do partograma para que seja possível diagnosticar as } \\
\text { possíveis alterações e indicar a tomada de condutas apropriadas para a sua resolução. }\end{array}$ \\
\hline A12 & Promover um ambiente tranquilo e aconchegante, visando propiciar a mulher uma experiência mais agradável. \\
\hline
\end{tabular}

Fonte: Autores.

O enfermeiro que irá auxiliar no parto humanizado deve ter em mente que a posição adequada, sempre será àquela referida pela parturiente, a que irá oferecer conforto e minimização da dor. São consideradas posições fisiológicas aquelas em que não há compressão de vasos sanguíneos e a progressão do parto vai a favor da gravidade (Cortês et al., 2018).

Dentre os benefícios da utilização das posições de cócoras, sentada ou quatro apoios pode-se citar à ajuda na passagem do bebê, porque contribui com a abertura vaginal e o encurtamento do canal de parto, acelerando o nascimento e a recuperação da mulher (Brasil, 2017)

Durante as fases de um parto há diversas condições que causam medo na parturiente, estas condições podem ser exemplificadas pela dor intensa, sensação de solidão, o processo de concepção do feto e a própria hospitalização. Entretanto, o 
desenvolvimento de orientações e de um plano de cuidados, por parte da enfermagem, acabam se tornando uma boa tática para a superação dessas dificuldades (Alves et al., 2018).

O enfermeiro exerce sua autonomia e posição de liderança ao assistir o parto. Dependendo da forma como este líder se comunica, isto pode influir positiva ou negativamente no empoderamento da mulher na parturição. Cabe ao enfermeiro esclarecer e fornecer segurança para a usuária do serviço, visando solucionar os medos ou angústias relatadas, desde o prénatal até o momento da concepção, se for o caso. É dever de toda a equipe que assiste ao parto, evitar práticas que inviabilize o parto e por consequência, que se consolide como uma violência obstétrica (Andrade et al., 2017).

\section{Conclusão}

A atenção adequada à mulher no momento do parto representa um passo indispensável, a fim de garantir que ela possa exercer a maternidade com confiança, segurança e bem-estar, o qual é um direito fundamental de toda mulher. A equipe de saúde deve estar preparada para acolher a gestante, seu companheiro e sua família, em especial o profissional enfermeiro, o qual deveria respeitar todos os significados desse momento e acrescentar confiança e segurança. Entre as atividades do enfermeiro, este deveria também minimizar a dor, ficar ao lado, dar conforto, esclarecer, orientar, enfim, ajudar a parir e a nascer. O cuidar humanizado deve estar centrado em uma assistência multiprofissional, tendo como foco principal a garantia do bem-estar da mulher e do recém-nascido, considerando os aspectos sociais, culturais e raciais.

Ressaltando a importância da assistência da equipe de enfermagem, a equipe tem o compromisso de mostrar opções de escolhas, são opções para o parto baseado em ciências e nos direitos que ela e seus familiares têm, na escolha de seu acompanhante, e sua posição durante o trabalho de parto. $\mathrm{O}$ acolhimento e a atenção oferecida pela equipe de enfermagem são de extrema importância para amenizar o tão temido medo do parto. A assistência realizada com métodos não farmacológicos e não invasivos contribuem positivamente para a efetivação do parto. Entre eles destaca-se a utilização do banho de chuveiro, da bola suíça, do uso do cavalinho, das barras, da deambulação e das massagens são benéficos para alívio das contrações e relaxamento da mulher, além de auxiliar na dilatação e expulsão do recém-nascido.

Sendo assim, a Assistência humanizada tem um papel fundamenta no trabalho de parto, que oferecer consiste em oferecer orientações, conforto, apoio emocional, bem-estar físico, segurança para parturiente. Entender e empregar as boas práticas de assistência, proporcionar ao processo de parturição uma assistência, com um número reduzido de intervenções, auxiliando no estímulo, respeito e na segurança do binômio mãe-filho na diminuição da morbimortalidade materna e neonatal.

Quando se pensa em conforto, a puérpera deve se sentir bem acolhida e respeitada pela equipe durante seu trabalho de parto, haverá um sentimento de satisfação, tendo a oportunidade de vivenciar um parto humanizado, onde ela será a protagonista daquele momento. De um modo geral, o ponto inicial para humanização da assistência é o respeito, considerado o fator determinante para haver uma contemplação do cuidado em todo o ciclo gravídico-puerperal. Neste sentido, a presente pesquisa poderá embasar novos artigos futuros que queiram preencher as lacunas, que por ventura, apresentem-se dentro deste contexto.

\section{Referências}

Alves, T. T. M. et al. (2018). Atuação da enfermeira obstetra no desenrolar do trabalho de parto e parto. Rev. enferm. atencao saude, 7(1), 41-50.

Andrade, L. F. B. de., Rodrigues, Q. P. \& Silva, R. de C. V. da. (2017). Boas Práticas na atenção obstétrica e sua interface com a humanização da assistência. Rev. enferm. UERJ. 25, e26442.

Bardin, L. Análise de conteúdo. Edições 70, 1977

Brasil. (2017). Ministério da Saúde. Portaria no. 2.436 de 21 de setembro de 2017. Diário Oficial da República Federativa do Brasil. 
Brasil. (2019). Ministério da Saúde. Sociedade Beneficente Israelita Brasileira Albert Einstein. Nota técnica para organização da rede de atenção à saúde com foco na atenção primária à saúde e na atenção ambulatorial especializada - saúde da mulher na gestação, parto e puerpério. Sociedade Beneficente Israelita Brasileira Albert Einstein. São Paulo: Ministério da Saúde.

Castro, J. \& Krapis, M. J. (2019). Parto humanizado na percepção das enfermeiras obstétricas envolvidas com a assistência ao parto. Revista Latino-Americana de Enfermagem, 13(6), 960-967.

Camillo, B. S. et al. (2016). Ações de educação em saúde na Atenção Primária a Gestantes e puérperas: revisão integrativa. Rev enferm ufpe, recife, 10(6), 4894-901.

Cortês, C. T. et al. (2018). Implementação das práticas baseadas em evidências na assistência ao parto normal. Rev. Latino-Am. Enfermagem. 26 , e2988.

Ercole, F. F., Melo, L. S. \& Alcoforado, C. L. C. (2014). Revisão integrativa versus revisão sistemática. Revista Mineira de Enfermagem, 18(1), 9-12.

Ferreira, J. B., Martinez, E. V. \& Chagas, A. C. F. (2018). Assistência de enfermagem no parto humanizado: uma revisão integrativa. Enfermagem Obstétrica. $5, \mathrm{e} 95$.

Fertonani, H. P., Pires, D. E. P., Biff, D. \& Scherer, M. D. A. (2015). The health care model: concepts and challenges for primary health care in Brazil. Ciência Saúde Coletiva, 20(6), 1869-78.

Freitas, P. F. et al. (2015). O parecer do Conselho Federal de Medicina, o incentivo à remuneração ao parto e as taxas de cesariana no Brasil. Cadernos de Saúde Pública, 31, 1839-1855.

Giantaglia, F. N. et al. (2017). O cuidado de enfermeiras de um programa de residência obstétrica sob o olhar da humanização. Rev. enferm. UFPE on line. 11(5), 1882-1891.

Leal, N. J. et al. (2018). Assistência ao pré-natal: depoimento das enfermeiras. Revista Cuidado é Fundamental, $10(1), 113122$.

Lehugeur, D., Strapasson, M. J. \& Fronza, E. (2017). Manejo não farmacológico de alívio da dor em partos assistidos por enfermeira obstétrica. Revista de Enfermagem UFPE on line. 11(12), 4929-4937.

Matos, G. C. de. et al. (2017). Grupos de gestantes: espaço para humanização do parto e nascimento. Revista De Pesquisa Cuidado é Fundamental Online, 9(2), 393-400

Melo, B. M. de. et al. (2017). Implementação das boas práticas na atenção ao parto em Maternidade de referência. Rev. Rene. 18(3), 376-82.

Pedroso, C. N. L. de S. \& López, L. C. (2017). À margem da humanização? Experiências de parto de usuárias de uma maternidade pública de Porto AlegreRS. Revista de Saúde Coletiva [online]. 27(4), 1163-1184.

Pereira, S. B. et al. (2018). Boas práticas de atenção ao parto e ao nascimento na perspectiva de profissionais de saúde. Rev Bras Enferm [Internet]. 71, 13939.

Moura, D. de A. et al. (2017). Assistência de enfermagem no parto humanizado. JOIN. Recuperado de: trabalho_ev081_md1_sa50_id1546_15092017172017.pdf (editorarealize.com.br)

Nascimento, F. C. V. do., Silva, M. P. da \& Viana, M. R. P. (2018). Assistência de enfermagem no parto humanizado. Rev Pre Infec e Saúde. 4, 6887. DOI: https://doi.org/10.26694/repis.v4i0.6887

Opas. OMS. Saúde Materna. 2018. https://www.paho.org/pt/node/63100\#: :text=Todos\%20os\%20dias\%2C\%20aproximadament e $\% 20830$,rurais\%20e\%20 comunidades $\% 20$ mais $\% 20$ pobres.

Possati, A. B. et al. (2017). Humanização do parto: significados e percepções de enfermeiras. Escola Anna Nery Revista de Enfermagem, 21(4), 1-6.

Santana, T. C. P. et al. (2019). Dificuldades dos enfermeiros no atendimento ao pré-natal de risco habitual e seu impacto no indicador de morbimortalidade materno-neonatal. Revista Eletrônica Acervo Saúde, 20, 1-11.

Santos, A. H. L. dos. et al. (2017). Práticas de assistência ao parto normal: formação na modalidade de residencia. Rev. enferm. UFPE on line. 11(1), 1-9.

Reis, C. S. C. dos. et al. (2016). Análise de partos acompanhados por enfermeiras obstétricas na perspectiva da desmedicalização. Rev. Pesqui. (Univ. Fed. Estado Rio J., Online). 8(4), 4972-4979.

Ursi, E. S. (2005). Prevenção de lesões de pele no perioperatório: revisão integrativa da literatura. Dissertação (Mestrado em Enfermagem) - Escola de Enfermagem, Universidade de São Paulo, 130. 